\title{
(6) OPEN ACCESS \\ Ethical issues raised by cluster randomised trials conducted in low-resource settings: identifying gaps in the Ottawa Statement through an analysis of the PURE Malawi trial
}

\author{
Tiwonge K Mtande, ${ }_{1}$ Charles Weijer, ${ }_{1}^{2}$ Mina C Hosseinipour, ${ }^{1,3}$ Monica Taljaard, ${ }^{4}$ \\ Mitch Matoga, ${ }^{1}$ Cory E Goldstein, ${ }^{5}$ Billy Nyambalo, ${ }^{6}$ Nora E Rosenberg ${ }^{1,3}$
}

${ }^{1}$ University of North Carolina Project, Lilongwe, Malawi ${ }^{2}$ Rotman Institute of Philosophy, University of Western Ontario, London, Ontario, Canada ${ }^{3}$ University of North Carolina at Chapel Hill, Chapel Hill, North Carolina, USA

${ }^{4}$ Ottawa Hospital Research Institute, Ottawa, Ontario, Canada

${ }^{5}$ Rotman Institute of Philosophy, Western University, London, Ontario, Canada

${ }^{6}$ Research Department, Ministry of Health Malawi, Lilongwe, Malawi

Correspondence to Dr Charles Weiier, Rotman Institute of Philosophy, Western University, London, ON N6A 5B8, Canada; cweijer@uwo.ca

Received 23 January 2019 Revised 16 April 2019 Accepted 27 April 2019 Published Online First 12 June 2019

\section{ABSTRACT}

The increasing use of cluster randomised trials in low-resource settings raises unique ethical issues. The Ottawa Statement on the Ethical Design and Conduct of Cluster Randomised Trials is the first international ethical guidance document specific to cluster trials, but it is unknown if it adequately addresses issues in low-resource settings. In this paper, we seek to identify any gaps in the Ottawa Statement relevant to cluster trials conducted in low-resource settings. Our method is (1) to analyse a prototypical cluster trial conducted in a low-resource setting (PURE Malawi trial) with the Ottawa Statement; (2) to identify ethical issues in the design or conduct of the trial not captured adequately and (3) to make recommendations for issues needing attention in forthcoming revisions to the Ottawa Statement. Our analysis identified six ethical aspects of cluster randomised trials in low-resource settings that require further guidance. The forthcoming revision of the Ottawa Statement should provide additional guidance on these issues: (1) streamlining research ethics committee review for collaborating investigators who are affiliated with other institutions; (2) the classification of lay health workers who deliver study interventions as health providers or research participants; (3) the dilemma experienced by investigators when national standards seem to prohibit waivers of consent; (4) the timing of gatekeeper engagement, particularly when researchers face funding constraints; (5) providing ancillary care in health services or implementation trials when a routine care control arm is known to fall below national standards and (6) defining vulnerable participants needing protection in low-resource settings.

\section{INTRODUCTION}

Cluster randomised trials (CRTs) are increasingly used to evaluate therapeutic interventions such as vaccines, as well as public health, health service delivery and knowledge translation interventions. CRTs are complex studies that present unique ethical challenges when compared with individually randomised controlled trials. ${ }^{1}$ In CRTs, the units of randomisation are intact social groups, or 'clusters', such as hospitals, neighbourhoods or medical practices, as opposed to individuals. ${ }^{2}$ Furthermore, the unit of randomisation, unit of intervention and unit of data collection may differ within the same study. ${ }^{2}$

To guide researchers and research ethics committees (RECs), Weijer et al developed a consensus document known as The Ottawa Statement on the Ethical Design and Conduct of CRTs. ${ }^{3}$ The Ottawa Statement has influenced other national and international research ethics policies. ${ }^{4}$ Although the Ottawa Statement is intended for use globally, ethical considerations arising in low-income and middle-income country (LMIC) settings are not comprehensively addressed. As the authors of the Ottawa Statement note, 'LMIC perspectives were under-represented (and)...we recommend that subsequent revisions include greater LMIC representation'. ${ }^{3}$ Indeed, little has been written about the ethical aspects of conducting CRTs in low-resource settings. ${ }^{6}$

In this paper, we seek to identify gaps in the Ottawa Statement relevant to CRTs conducted in low-resource settings. Our method is (1) to analyse a prototypical CRT conducted in a low-resource setting (PURE Malawi trial) with the Ottawa Statement; (2) to identify ethical issues in the design or conduct of the trial not captured adequately and (3) to make recommendations for issues needing attention in forthcoming revisions to the Ottawa Statement. The study team involved authors of the Ottawa Statement and researchers involved in the design and conduct of the PURE Malawi trial.

\section{PURE MALAWI TRIAL}

In 2011, the Malawi Ministry of Health introduced a novel strategy to improve the prevention of mother-to-child transmission of HIV, called Option $\mathrm{B}+$. The Option $\mathrm{B}+$ programme recommends lifelong antiretroviral therapy to all pregnant and breastfeeding women living with HIV—regardless of their CD4 count level or WHO clinical stageand 6 weeks of daily nevirapine for the newborn infant. Although the strategy resulted in an increase in antiretroviral therapy initiation, uptake and retention were suboptimal among asymptomatic pregnant and breastfeeding women due to low treatment literacy, stigma and the lack of psychosocial support. ${ }^{89}$

The PURE Malawi trial was a parallel-arm CRT that evaluated whether two novel peer-support interventions (health facility-based or community-based peer support) compared with routine care could improve uptake and retention of mothers and infants in Malawi's Option B+ programme. Twenty-one health facilities were stratified by size and 
randomly allocated to one of three study arms: routine care as defined by national standards; health facility-based peer support; or community-based peer support. Medical personnel invited pregnant and breastfeeding women to participate if they were 15 years or older, HIV-positive and antiretroviral therapy naïve. The primary outcome was the proportion of women retained alive and on antiretroviral therapy at 12 months post-treatment initiation. Secondary outcomes included the proportion of women on antiretroviral therapy at 24 months, partner and child HIV testing, impact of task shifting on health facilities and cost-effectiveness.

At health facilities randomised to routine care, women received care according to Malawi's HIV Clinical Guidelines. ${ }^{10}$ At facilities randomised to facility-based peer support, women received routine care and met with 'mentor mothers'. Mentor mothers are HIV-positive mothers who have gone through the prevention of mother-to-child transmission (PMTCT) process, and were recruited and trained by investigators. They provided one-on-one support to women at each clinic visit, led weekly clinic-based support groups and contacted women within one week of a missed appointment. ${ }^{11}$ At facilities randomised to community-based peer support, women received routine care and met with 'expert mothers'. Expert mothers are HIV-positive mothers who have gone through the PMTCT process, and were recruited and trained by investigators. They conducted home visits to participants to provide HIV education, provided clinic visit reminders and led monthly community-based support group meetings. ${ }^{11}$ Male partners of the enrolled women were invited to participate. The main data sources were routine adult and infant patient care cards and clinic registers. Additionally, mentor and expert mothers documented the timing and nature of each encounter with women.

Of the 1361 eligible women approached, 1272 women provided written consent to participate in the PURE Malawi trial. The women were given information describing the trial's purpose and the arm to which their facility was allocated. Women consented to interventions and data collection.

Phiri et al reported that antiretroviral treatment uptake was higher in facility-based models (86\%; relative difference (RD): $6 \%, 95 \%$ CI: $-3 \%$ to $15 \%)$ and community-based models (90\%; RD: $9 \%, 95 \%$ CI: $1 \%$ to $18 \%)$ compared with routine care $(81 \%)$, but not statistically significant. ${ }^{11}$ At 24 months, retention was significantly higher in the facility-based peer-support arm (80\%; RD: $13 \%, 95 \%$ CI: $1 \%$ to $26 \%$ ) and community-based peer-support arm (83\%; RD: $16 \%$, 95\% CI: $3 \%$ to $30 \%)$ compared with routine care $(66 \%)$. Viral suppression was similar across the arms at 6 and 24 months among those retained in care. Overall, the trial concluded that peer-support interventions improved uptake of the Option B+ programme and retention at 24 months.

\section{IDENTIFYING GAPS IN THE OTTAWA STATEMENT THROUGH AN ANALYSIS OF THE PURE MALAWI TRIAL}

The Ottawa Statement provides 15 recommendations for CRTs. These fall into domains: justification for cluster randomisation; REC review; identification of research participants; informed consent procedures; the role and authority of gatekeepers; benefit-harm analysis; and the protection of vulnerable participants. ${ }^{3}$ Domains and recommendations were developed after extensive review and consensus reached by stakeholders experienced with CRTs. In what follows, we analyse the PURE Malawi trial using these domains to identify gaps between guidance in the Ottawa Statement and a CRT conducted in a low-resource setting. These gaps inform our recommendations for issues needing further attention (table 1).

\section{Justification for CRT design}

CRTs always require more participants than an individually randomised trial because the outcomes of participants within

Table 1 Recommendations for issues needing attention in forthcoming revisions to the Ottawa Statement

\begin{tabular}{|c|c|}
\hline Ethical domain & Issues requiring further guidance \\
\hline $\begin{array}{l}\text { Justification for cluster } \\
\text { randomised trial (CRT) } \\
\text { design }\end{array}$ & No change required. \\
\hline $\begin{array}{l}\text { Research ethics } \\
\text { committee (REC) review }\end{array}$ & $\begin{array}{l}\text { Make explicit the need for host and sponsor country review in low-resource settings. } \\
\text { Clarify when home institution REC review is required for investigators in specific roles. Investigators whose role is limited to protocol development } \\
\text { or analysis likely do not require such review. } \\
\text { Multiple REC reviews are inefficient. Are there further means to streamline study review by RECs while ensuring adequate protections for research } \\
\text { participants in low-resource settings? }\end{array}$ \\
\hline $\begin{array}{l}\text { Identifying research } \\
\text { participants }\end{array}$ & $\begin{array}{l}\text { Lay health workers are commonly involved in CRTs in low-resource settings. When should they be considered research participants? } \\
\text { Broadly, guidance is required on when health providers who deliver the study intervention are also targeted by the intervention and should be } \\
\text { considered participants. }\end{array}$ \\
\hline $\begin{array}{l}\text { Obtaining informed } \\
\text { consent }\end{array}$ & $\begin{array}{l}\text { Address conflicts with national laws and guidelines with regard to waiver of consent. } \\
\text { Encourage lawmakers and ethics bodies to adopt provisions to allow for waiver of consent for CRTs. } \\
\text { When host and sponsor country REC reviews are required, the host country REC should undertake the primary responsibility in approving a waiver } \\
\text { of consent. }\end{array}$ \\
\hline Gatekeepers & $\begin{array}{l}\text { Guidance on the timing of gatekeeper engagement is required. In low-resource settings, the CRT may need to be funded before extensive } \\
\text { gatekeeper involvement in feasible. } \\
\text { Gatekeeper roles may be diverse and include involvement in protocol design enrolment, implementation and reporting. Role-specific guidance on } \\
\text { gatekeeper involvement is needed. }\end{array}$ \\
\hline $\begin{array}{l}\text { Assessing benefits and } \\
\text { harms }\end{array}$ & $\begin{array}{l}\text { Further guidance is required on the appropriate control conditions in CRTs in low-resource settings. } \\
\text { When CRTs seek to evaluate implementation or health services interventions designed to improve care delivery, usual care as delivered ought to be } \\
\text { deemed acceptable even if it falls below national care standards. } \\
\text { Further guidance is needed on the responsibility of multiple stakeholders in sustaining effective interventions after CRT completion in low-resource } \\
\text { settings. }\end{array}$ \\
\hline $\begin{array}{l}\text { Protecting vulnerable } \\
\text { participants }\end{array}$ & $\begin{array}{l}\text { Adopt a view of vulnerable persons as people who 'may have an increased likelihood of being wronged'. The emphasis this places on contextual } \\
\text { factors is relevant to low-resource settings. }\end{array}$ \\
\hline
\end{tabular}


the same cluster are correlated with one another. ${ }^{2}$ CRTs are also more prone to bias, including baseline imbalances between clusters, recruitment bias, performance bias and attrition bias. ${ }^{12} 13$ Accordingly, the Ottawa Statement requires that researchers explicitly justify the use of a cluster-randomised design.

Analysis of PURE Malawi trial. In the PURE Malawi trial, several factors supported the cluster-randomised design. ${ }^{14}$ First, as the interventions involve peer support, an individually randomised design would have been prone to contamination, as control and intervention patients would interact. Second, the recruitment of mentor and expert mothers involved training, and it was convenient and less costly to conduct training only in intervention institutions. Third, it may have been perceived as unfair to prevent women from receiving peer support conducted within their own health facility or community. Finally, there was need for a pragmatic evaluation to assess the effectiveness of peer-support strategies as they would be delivered in practice. Therefore, the use of a cluster-randomised design in the PURE Malawi trial is justified.

Gaps/recommendations for the Ottawa Statement. None.

\section{Research ethics committee review}

All research involving human participants must be reviewed by a REC. The Ottawa Statement concludes that all health-related CRTs, including those with quality improvement, health services or implementation interventions, are research involving human participants and, accordingly, must be reviewed by a REC. ${ }^{3}$ Other international documents require that research which is externally funded and conducted in low-resource settings be approved by a REC in both host and sponsor countries. ${ }^{4}$

Analysis of PURE Malawi trial. Malawi's National Health Sciences Research Committee (host country), the University of North Carolina Institutional Review Board (US sponsor), the University of Toronto Research Ethics Board (Canadian sponsor) and the WHO Ethics REC (international sponsor) approved the PURE Malawi trial through full board reviews. ${ }^{11}$ The PURE Malawi trial was designed and implemented by the PURE Malawi Consortium, whose members fulfilled various roles in the project. All members involved in the recruitment and follow-up of participants sought or were 'covered' by approvals from their home institution's REC. Thus, all required REC approvals were obtained.

Gaps/recommendations for the Ottawa Statement. The Ottawa Statement is silent on the need for host country and sponsor country review when a CRT is conducted in a low-resource setting. Additional guidance on when a protocol must be submitted for sponsor country review is needed. Following what happened in the PURE Malawi trial, is home institution REC approval necessary for investigators at other institutions, if host and sponsor countries have approved the study? If so, does it matter what role the investigator is playing in the study? For instance, if an investigator's role is limited to protocol development or the analysis of study data, must approval from her institution be sought? Too many full board REC reviews add needless burdens to CRTs. ${ }^{15}$ Minimising the number of full board REC reviews required (perhaps to a single host and sponsor REC), consistent with adequate protections for participants, would promote the efficient conduct of CRTs.

\section{Identifying research participants}

In CRTs, different groups of people may be affected by different parts of the study. Thus, identifying research participants in CRTs may not be straightforward. The Ottawa Statement defines a research participant as 'an individual whose interests may be affected as a result of study interventions or data collection procedures'. ${ }^{3}$ This definition is operationalised by four criteria, any one of which is sufficient. A research participant is someone: (1) who is the recipient of the study or control intervention; (2) who is the direct target of an environmental manipulation; (3) with whom researchers interact for study purposes or (4) about whom identifiable private information is obtained. ${ }^{3}$

Analysis of PURE Malawi trial. HIV-positive pregnant or breastfeeding women eligible for Option $\mathrm{B}+$ in participating centres ought to be considered research participants. The peer-support interventions targeted these women (criterion 1). Furthermore, women interacted with researchers for study purposes (criterion 3), and their data on missed appointments and attendance at support group meetings were recorded (criterion 4). Finally, their routinely collected health data, including clinical visits and HIV viral load, were obtained (criterion 4).

The male partners of participating women are research participants. They were invited to participate in the study and encouraged to undergo HIV testing (criterion 1), and their test results were accessed (criterion 4). The newborn children of women participating in the study are also research participants, as their routinely collected health data, including HIV test results, were accessed (criterion 4).

Gaps/recommendations for the Ottawa Statement. More guidance is required to determine if lay workers involved in the delivery of health interventions ought to be considered research participants. When health workers merely deliver the study intervention, they are not research participants; when they are the intended recipients of the intervention, however, they are research participants. Difficult cases lie in the middle. Consider the role of the mentor and expert mothers in the PURE Malawi trial. They delivered peer support to pregnant and breastfeeding women in the trial. But, they were recruited and trained by the investigators for this role, and one of the study objectives examined the impact of task shifting on health facilities. Health services and implementation CRTs commonly train health providers to deliver study interventions. More guidance is needed on the proper classification of health workers as research participants in CRTs.

\section{Obtaining informed consent}

Informed consent is a central protection for research participants. The Ottawa Statement says: 'Researchers must obtain informed consent from human research participants in a CRT, unless a waiver of consent is granted by a REC'. ${ }^{3}$ A REC may approve a waiver of consent when (1) the trial is not feasible if informed consent is required and (2) study participation poses no more than minimal risk. ${ }^{3}$ Provided they pose only minimal risk, interventions delivered to the cluster as a whole commonly qualify for a waiver of consent; as all cluster members will receive the intervention, refusal of consent in such cases is meaningless. A waiver of consent should not be used solely for one study arm as it may lead to differential recruitment across arms. This can seriously undermine study validity as outcomes may be driven by baseline health differences between groups rather than the study interventions. The Ottawa Statement urges researchers to get consent where possible: 'If obtaining informed consent is feasible for some but not all study interventions or data collection procedures, then researchers should obtain separate informed consent, where possible, for each procedure. ${ }^{3}$

Analysis of PURE Malawi trial. HIV-positive pregnant or breastfeeding women who had been screened for eligibility were invited to participate by a trained clinical staff member. ${ }^{11}$ The consent document explained the trial's purpose, the intervention 
to which their health facility had been randomised, data collection procedures and the risks and benefits of participation. Women were asked to provide consent on behalf of their infants. This recruitment procedure was used across all randomised health facilities. Women who provided written informed consent were enrolled. Of the 1756 women who were screened for eligibility, 395 were deemed ineligible (primarily because they were already on antiretroviral treatment). ${ }^{11}$ Of the 1361 eligible women approached for consent, 1272 (93.4\%) agreed to participate. The acceptance rates were similar among the facility intervention $(92.6 \%)$, community intervention $(97.9 \%)$ and routine care arms (90\%). Written informed consent was also sought from male partners of the enrolled women.

In the PURE Malawi trial, consent could have been handled differently. Following the guidance of both the Council for International Organizations of Medical Sciences (CIOMS) and the Ottawa Statement, a waiver of consent for study interventions would have been permissible. Peer-support interventions in health facilities and communities were integrated into the care received by all HIV-positive pregnant or breastfeeding women. While women could have chosen not to attend peer-support group meetings, they nonetheless would have been exposed to one-on-one peer support, home visits and follow-up for missed visits. As a result, women who declined participation were nonetheless exposed to the interventions (although their data were not included in the analysis). As peer support has been studied in other contexts and found to be safe and efficacious, it poses no more than minimal risk to participants. ${ }^{16}{ }^{17}$ One consequence of a waiver of consent is that notification of the ongoing trial (eg, posters in health clinics) would be required.

There is, however, an impediment to the use of a waiver of consent in Malawi. Malawian RECs do not allow a waiver of consent for studies involving identifiable participants. They base this on section 19(5) of the Constitution of Malawi: 'No person shall be subjected to medical or scientific experiment without his or her consent'. ${ }^{18}$ How should investigators proceed when national standards seem to prohibit waivers of consent?

Gaps/recommendations for the Ottawa Statement. The Ottawa Statement should address conflicts between its recommendations and relevant national law and guidelines on waivers of consent. Resolving these conflicts will be essential to obtain waivers of consent for cluster-level interventions. Furthermore, the Ottawa Statement is silent on whether multiple REC approvals are needed for a waiver of consent. We suggest the host country REC should approve waivers of consent and notify sponsor country RECs reviewing the CRT.

\section{Gatekeepers}

The Ottawa Statement defines gatekeepers as individuals or bodies who may be called on to protect the group-based interests that are affected by enrolment in a CRT ${ }^{3}{ }^{3}$ Gatekeepers may include directors of health facilities, community leaders or government officials. While each has a role in protecting group interests, the Ottawa Statement mandates that gatekeepers should not provide consent on behalf of cluster members. When CRT enrolment may substantially affect group interests, researchers should obtain the gatekeeper's permission for group enrolment. Moreover, researchers should seek to protect group interests through consultations to inform study design, conduct and reporting. ${ }^{3}$

Analysis of PURE Malawi trial. Gatekeeper permission was obtained at multiple levels, although at different times. The Malawi Ministry of Health was a member of the PURE Malawi Consortium; accordingly, it was involved in the trial's design and provided permission at the national level. After the trial was funded, permission for enrolment and implementation was sought from local gatekeepers. Permission was obtained from 7 health districts responsible for 18 of the health facilities. Separate permission was obtained from two hospitals and a clinic that fell outside the aegis of the health districts.

Permission was also obtained from community leaders within the catchment areas of the health facilities. Village heads were invited to a meeting to be informed about the trial and discuss implementation and barriers. Important changes were made to the PURE Malawi trial at this stage. For instance, rather than interacting only with HIV-positive mothers, it was agreed that peer-support workers reach out to all pregnant or breastfeeding women by integrating elements of safe motherhood programmes. In this way, confidentiality protections for HIV-positive mothers were enhanced.

Gaps/recommendations for the Ottawa Statement. The Ottawa Statement recognises the need to engage multiple levels of gatekeepers. More guidance is needed on the timing of gatekeeper engagement. Practically, the trial may need to be funded before extensive engagement can occur. Furthermore, gatekeeper involvement may differ in protocol design, enrolment, implementation or reporting. More guidance on which gatekeepers to include in each of these roles would be helpful. Finally, we recommend that the Ottawa Statement take on the language of 'community engagement', as it encompasses community involvement, consultation and partnership. ${ }^{19}$

\section{Assessing benefits and harms}

The Ottawa Statement sets out three recommendations for analysing the benefits and harms of CRTs. First, researchers must ensure that the study interventions are adequately justified, and that there is evidence to support the claim that the interventions are 'consistent with competent practice in the field of study relevant to the $\mathrm{CRT}^{\prime}{ }^{3}$ Second, researchers must adequately justify the choice of the control intervention. When the control arm is routine care, 'individuals in the control arm must not be deprived of the effective care or programmes to which they would have access, were there no trial'. ${ }^{3}$ Third, and finally, researchers must ensure that the risks of data collection procedures are minimised consistent with sound scientific design, and reasonable in relation to the knowledge gained. ${ }^{3}$ The Ottawa Statement requires that, when reviewing study protocols, the REC consider whether and when the control clusters will receive the study intervention if it is shown to be effective. ${ }^{3}$

Analysis of PURE Malawi trial. The two study interventions were justified. In Malawi, since 2009, a project called Mothers2Mothers has been providing facility-based peer support to HIV-positive women in select sites. Another Malawian study showed that shifting tasks to lay community health workers improved antiretroviral therapy retention and service delivery. ${ }^{20}$ A pilot initiative in Malawi's Zomba district showed that the identification and involvement of 'expert patients' can improve treatment uptake and the tracing of clients on antiretroviral therapy. ${ }^{17}$ Yet, without a rigorously conducted trial, it was unknown whether the addition of facility-based or community-based peer support to the Option B+ programme would be effective or affordable. ${ }^{11}$

The justification of the control arm in the PURE Malawi trial is complex. Participants in the routine care arm received HIV care in accordance with Malawian Ministry of Health Option $\mathrm{B}+$ guidelines. ${ }^{11}$ According to these guidelines, women who do not attend the clinic after 60 days of a missed appointment are to be traced, contacted and encouraged to return to the clinic. 
Unfortunately, tracing rarely occurs and the quality of care varies across facilities. ${ }^{14}$ Transport, staffing and resource shortages all contribute to quality-of-care challenges. ${ }^{21}$

Was the routine care control arm in the PURE Malawi trial justified? Recall that the Ottawa Statement requires that participants in the control arm not be deprived of 'programmes to which they would have access' ${ }^{3}$ On one reading of this requirement, the control arm is justified. Had the PURE Malawi trial not been conducted, women attending control facilities would have received the same care and follow-up. Thus, they were not denied access to existing programmes.

Another reading of the requirement leads to a different conclusion. London draws a distinction between two different types of local standard of care: de facto and de jure. ${ }^{22}$ The de facto standard of care is the care actually delivered in local facilities; the de jure standard is the care that should be delivered as defined by national policy. London argues that the control arms ought to be held to the latter standard, as the former allows inefficiencies in care delivery to justify exploitation. As the PURE Malawi trial did not add resources to strengthen care in control facilities, the control arm was not justified.

How should the PURE Malawi trial investigators have proceeded? One option was to add resources to control facilities to ensure women received follow-up as defined by Option $\mathrm{B}+$. As the trial sought to determine whether the addition of peer support would improve adherence and retention of women receiving antiretroviral treatment, this would have undermined the trial's validity. Another option was to exclude the worst performing facilities, enrolling only those centres with reasonably good performance. But this would have undermined the trial's generalisability, as the results would not be applicable to all facilities. A third option was what the researchers did: address the efficiency and affordability of peer support under real-world conditions. The result of the study as designed seems most informative to health system managers.

Given the limited funding the researchers could not guarantee access to peer support after the trial's completion. Instead, they provided the study results to the Ministry of Health and advocated for the peer-support programmes.

Gaps/recommendations for the Ottawa Statement. Further guidance is required on the appropriate selection of control conditions in trials that seek to improve delivery of care defined by national policies. We suggest that when implementation or health services CRTs are seeking to improve treatment delivery and affordability, that control conditions should be permitted to violate the de jure standard so long as they pass the de facto standard.

Ensuring access to effective study interventions would mitigate concerns about care received in the control arm. Post-trial access in low-resource setting remains a critical issue. Most often CRT designs are employed in health systems and implementation research, therefore, post-trial access ought to be planned before study commencement. Further guidance from the Ottawa Statement should define the role of sponsors and other parties in low-resource settings in sustaining effective interventions past the completion date of the trial.

\section{Protecting vulnerable participants}

Vulnerable participants require additional protections. The Ottawa Statement defines vulnerable participants as (1) children, (2) incapable adults, (3) people at undue risk of harm as a result of study participation and (4) people in subordinate positions within social or organisational structures. ${ }^{3}$ The Ottawa Statement includes two recommendations for research involving vulnerable participants. First, it cautions that clusters may contain 'hidden' vulnerable participants, and researchers should be careful to ensure these individuals receive needed protections. Second, when cluster members are part of a hierarchy, such as employees within a healthcare institution, special attention must be paid to 'recruitment, privacy and consent procedures for those participants'.

Analysis of PURE Malawi trial. Due to prevalent HIV stigma and discrimination, all participants in the PURE Malawi trial were vulnerable. As a result, researchers and gatekeepers agreed to provide additional measures to protect confidentiality. Regardless of HIV status the mentor mothers and expert mothers approached every pregnant or breastfeeding woman in the facility and community.

Gaps/recommendations for the Ottawa Statement. More recent ethical guidance has moved away from classifying groups as vulnerable, and focuses on individual characteristics and contextual factors. For instance, CIOMS defines vulnerable persons as those who 'may have an increased likelihood of being wronged'. Broadly, there are three types of wrongs: autonomy wrongs (eg, failing to identify decisionally incapable participants); welfare wrongs (eg, failing to protect participants who are at greater risk) and justice wrongs (eg, exposing the socially marginalised to greater stigmatisation). ${ }^{23}$ The emphasis on contextual factors is particularly relevant in low-resource settings, as individuals may be vulnerable simply because they are poor, illiterate or have inadequate access to healthcare. An updated version of the Ottawa Statement should adopt this approach to vulnerability because of its relevance to low-resource settings.

\section{CONCLUSION}

Our analysis of the PURE Malawi trial using the Ottawa Statement highlighted a number of issues not addressed adequately in the Ottawa Statement (table 1). Many issues identified are specific to CRTs conducted in low-resource settings; others apply to all settings. When revised, the Ottawa Statement should address these issues and provide needed guidance for CRTs in low-resource settings. Future work should examine other CRTs conducted in low-resource settings to identify if the same ethical issues (or new issues) are raised.

Acknowledgements The authors would like to thank the two peer reviewers for their most helpful comments. The authors would also like to thank Anthony Belardo for editing the final draft of the paper.

Contributors TKM and CW conceptualised the paper. TKM wrote the first draft, and CW edited and rewrote sections of the next draft. All other authors provided comments on subsequent drafts of the paper. All authors approved the final paper.

Funding TKM is a SARETI scholar funded by a Global Forum on Bioethics in Health Research fellowship, Wellcome Trust grant number 211061/Z/18/Z. This work is also supported by the Canadian Institutes of Health Research through the Project Grant competition (competitive, peer-reviewed), award number PJT-153045. CW holds the Canada Research Chair in Bioethics at Western University. NER is supported by the National Institutes of Health through R00 MH104154 and P30 Al50410.

Competing interests CW receives consulting income from Eli Lilly and Company Canada.

Patient consent for publication Not required.

Provenance and peer review Not commissioned; externally peer reviewed.

Data sharing statement This article is argument-based, so there are no unpublished data to be shared.

Open access This is an open access article distributed in accordance with the Creative Commons Attribution Non Commercial (CC BY-NC 4.0) license, which permits others to distribute, remix, adapt, build upon this work non-commercially, and license their derivative works on different terms, provided the original work is properly cited, appropriate credit is given, any changes made indicated, and the use is non-commercial. See: http://creativecommons.org/licenses/by-nc/4.0/. 


\section{REFERENCES}

1 Weijer C, Grimshaw JM, Taljaard M, et al. Ethical issues posed by cluster randomized trials in health research. Trials 2011;12:100.

2 Donner A, Klar N. Pitfalls of and controversies in cluster randomization trials. Am J Public Health 2004;94:416-22.

3 Weijer C, Grimshaw JM, Eccles MP, et al. The Ottawa Statement on the Ethical Design and Conduct of Cluster Randomized Trials. PLoS Med 2012;9:e1001346.

4 Council for International Organizations of Medical Sciences (CIOMS). International Ethical Guidelines for Health-related Research involving Humans. Geneva, Switzerland: Council for International Organizations of Medical Sciences, 2016.

5 U.S. Secretary's Advisory Committee on Human Research Protections. Recommendations on Regulatory Issues in Cluster Studies. 2019. https://www.hhs. gov/ohrp/sachrp-committee/recommendations/2014-july-3-letter-attachment-c/index. html

6 Gopichandran V, Luyckx VA, Biller-Andorno N, et al. Developing the ethics of implementation research in health. Implement Sci 2016:11:161.

7 Osrin D, Azad K, Fernandez A, et al. Ethical challenges in cluster randomized controlled trials: experiences from public health interventions in Africa and Asia. Bull World Health Organ 2009;87:772-9.

8 Haas AD, Msukwa MT, Egger M, et al. Adherence to antiretroviral therapy during and after pregnancy: cohort study on women receiving care in Malawi's Option B+ Program. Clin Infect Dis 2016;63:ciw500-1235.

9 Tenthani L, Haas AD, Tweya $H$, et al. Retention in care under universal antiretroviral therapy for HIV-infected pregnant and breastfeeding women ('Option B+') in Malawi. AIDS 2014;28:589-98.

10 Ministry of Health. Malawi clinical management of hiv in children and adults. Malawi: Ministry of Health, 2011.

11 Phiri S, Tweya $\mathrm{H}$, van Lettow $\mathrm{M}$, et al. Impact of facility- and community-based peer support models on maternal uptake and retention in Malawi's Option B+ HIV Prevention of Mother-to-Child Transmission Program: A 3-Arm Cluster Randomized
Controlled Trial (PURE Malawi). J Acquir Immune Defic Syndr 2017;75(Suppl 2):S140-S148

12 Eldridge S, Kerry S, Torgerson DJ. Bias in identifying and recruiting participants in cluster randomised trials: what can be done? BMJ 2009:339:b4006.

13 Giraudeau B, Ravaud P. Preventing bias in cluster randomised trials. PLoS Med 2009;6:e1000065.

14 Rosenberg NE, van Lettow M, Tweya $\mathrm{H}$, et al. Improving PMTCT uptake and retention services through novel approaches in peer-based family-supported care in the clinic and community: a 3-arm cluster randomized trial (PURE Malawi). J Acquir Immune Defic Syndr 2014;67(Suppl 2):S114-S119.

15 Simmons D, Bunn C, Nakwagala F, et al. Challenges in the Ethical Review of Peer Support Interventions. Ann Fam Med 2015;13(Suppl 1):S79-S86.

16 Baek C, Mathambo V, Mkhize S, et al. Key findings from evaluation of the Mothers2Mothers program in KwaZulu-Natal, South Africa: Horizons Final Report. Washington, DC: Population Council, 2007.

17 Gawa L, Singogo E, Chan AC, et al. Evaluation of an expert patient program for PMTCT services at a peri-urban health centre in Zomba District, Malawi. Poster Presentation, IAS, Rome, 2011.

18 Constitution of the Republic of MalawiWebsite. 1996. http://www.wipo.int/edocs/ lexdocs/laws/en/mw/mw002en.pdf (accessed 7 Apr 2019).

19 Dresser R. A Deep Dive into Community Engagement. Narrat Inq Bioeth 2017:7:41-5

20 Bemelmans $\mathrm{M}$, van den Akker T, Ford N, et al. Providing universal access to antiretroviral therapy in Thyolo, Malawi through task shifting and decentralization of HIVIAIDS care. Trop Med Int Health 2010;15:1413-20.

21 Tweya H, Gugsa S, Hosseinipour M, et al. Understanding factors, outcomes and reasons for loss to follow-up among women in Option B+ PMTCT programme in Lilongwe, Malawi. Trop Med Int Health 2014;19:1360-6.

22 London AJ. The ambiguity and the exigency: clarifying 'standard of care' arguments in international research. J Med Philos 2000:25:379-97.

23 Hurst SA. Vulnerability in research and health care; describing the elephant in the room? Bioethics 2008:22:191-202. 